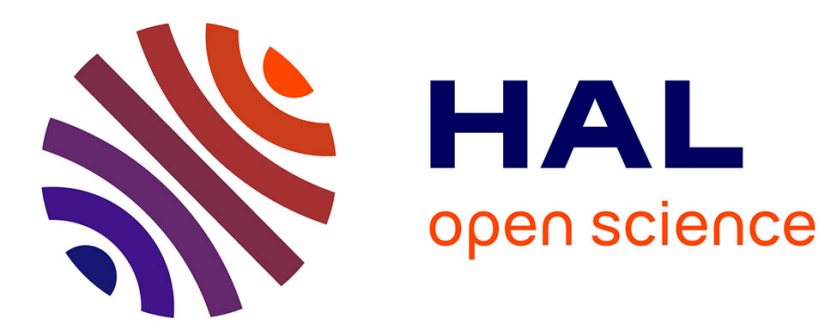

\title{
TRAITEMENT DE L'EFFET DOPPLER EN ACOUSTIQUE PASSIVE
}

\author{
J. de Reffye
}

\section{To cite this version:}

J. de Reffye. TRAITEMENT DE L'EFFET DOPPLER EN ACOUSTIQUE PASSIVE. Journal de

Physique IV Proceedings, 1992, 02 (C1), pp.C1-999-C1-1002. 10.1051/jp4:19921219 . jpa-00251186

\section{HAL Id: jpa-00251186 https://hal.science/jpa-00251186}

Submitted on 1 Jan 1992

HAL is a multi-disciplinary open access archive for the deposit and dissemination of scientific research documents, whether they are published or not. The documents may come from teaching and research institutions in France or abroad, or from public or private research centers.
L'archive ouverte pluridisciplinaire HAL, est destinée au dépôt et à la diffusion de documents scientifiques de niveau recherche, publiés ou non, émanant des établissements d'enseignement et de recherche français ou étrangers, des laboratoires publics ou privés. 


\title{
TRAITEMENT DE L'EFFET DOPPLER EN ACOUSTIQUE PASSIVE
}

\author{
J. de REFFYE
}

Société CR2A, 19 Avenue Dubonnet, F-92411 Courbevoie cedex, France

\begin{abstract}
On cherche à utiliser la variation de fréquence observée sur les raies LOFAR en acoustique passive comme on utilise la différence de fréquence observée en sonar actif. Dans ce cas, la fréquence reçue peut être comparée à la fréquence émise, ce qui n'est pas le cas en sonar passif. C'est pourquoi, dans ce cas, on cherche à utiliser la dérivée temporelle de la fréquence qui, elle, ne nécessite pas d'information a priori. Son traitement permet une meilleure détection des raies instables en fréquence qui apparaissent souvent en analyse LOFAR. Associé à une normalisation de type TFAC, un tel traitement a été comparé au traitement LOFAR actuellement utilisé en acoustique sous-marine.
\end{abstract}

\section{INTRODUCTION}

Le traitement actuel des signaux LOFAR est essentiellement orienté vers la visualisation des raies, c'est-à-dire qu'il vise à augmenter localement le contraste pour les faire ressortir sur l'écran de visualisation (1). Les résultats sont d'autant meilleurs que la raie est pure, c'est-à-dire qu'elle est issue d'un signal cohérent. Mais il reste le problème de la référence de bruit qui n'est pratiquement pas abordée dans le traitement actuel, car la détection finale s'effectue essentiellement de manière visuelle. La méthode proposée vise à exploiter au maximum la cohérence des raies par rapport à celle du bruit en utilisant l'espace (fréquence, dérivée temporelle de la fréquence) à 2 dimensions, au lieu de l'espace fréquentiel à une dimension. 
On a d'abord cherché à utiliser des traitements non linéaires mais les résultats ont toujours été mauvais, à cause du mauvais rapport signal à bruit, sauf si l'on utilise un traitement non linéaire en post-intégration. On a défini le principe d'un récepteur TFAC sur 2 paramètres, suivi d'une postintégration.

\section{PRNCIPE DE LA METHODE}

On a cherché, avant tout, à rester compatible avec les méthodes existantes. En particulier, on a respecté la visualisation LOFAR existante si bien que l'on peut utiliser l'algorithme proposé ici dans les mêmes conditions que l'algorithme actuellement utilisé.

L'algorithme proposé réalise une normalisation sur la fréquence et sa première dérivée temporelle (par TFAC fréquentiel et variation de fréquence) et une détection du signal sur la variation de fréquence correspondant au maximum de niveau.

On propose 2 réglages différents de l'algorithme selon que l'on veut détecter des "raies fines" ou des "raies larges et floues".

D'une manière générale, on effectue la calcul suivant:

$s(\Delta t)$ $s(M \Delta t), s(M+1) \Delta t)$ $s(2 M \Delta t), s(2 M+1) \Delta t)$ $s(3 M \Delta t)$
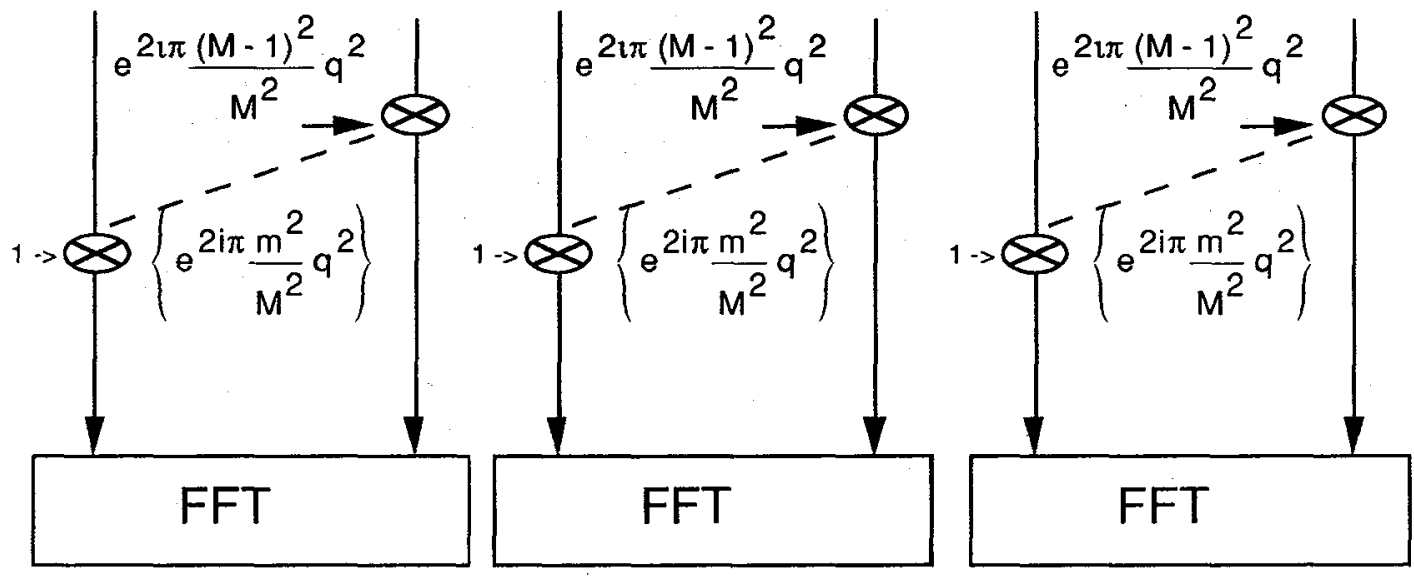

Une première opération corrige la variation linéaire de fréquence. La FFT, ensuite, corrige la variation linéaire de phase. On a pris 3 blocs pour réaliser une post-intégration. 
En sortie de FFT, on propose 2 traitements, l'un est adapté au raies stables, destinée à les affiner en vue de mesures précises, l'autre est adaptée aux raies larges et floues, en vue de les intégrer correctement.

\subsection{Traitement d'affinage - Doppler}

Dans ce traitement, on sépare les raies du bruit par la fréquence et sa dérivée : on calcule, après FFT, pour une fréquence $v_{o} \widetilde{x}(q, v, n)$ représentant la sortie FFT à la fréquence $v$ et de pente $q$, du $n{ }^{\text {ème }}$ bloc.

$$
\sum_{n=1}^{3}\left|\tilde{x}\left(0, v_{0}, n\right)\right|^{2}
$$

$$
\overline{\sum_{n=1}^{3}\left|\tilde{x}\left(-1, v_{0} \pm 3, n\right)\right|^{2}+\left|\tilde{x}\left(+1, v_{0} \pm 3, n\right)\right|^{2}+\left|\tilde{x}\left(-2, v_{0} \pm 3, n\right)\right|^{2}+\left|\tilde{x}\left(+2, v_{0} \pm 3,\right)\right|^{2}}
$$

Les résultats sur des signaux réels sonar du traitement sont donnés à la figure 1.b, la figure 1.a étant le traitement actuel adapté à ce cas de figure.

\subsection{Traitement sur raies larges et floues}

II s'agit de raies pouvant avoir de fortes variations de fréquence. On effectue avec les mêmes notations le traitement suivant :

$$
\operatorname{Max}-2 \leq q_{0} \leq+2 \frac{\sum_{n=1}^{3}\left|\tilde{x}\left(q_{0}, v_{0}, n\right)\right|^{2}}{\sum_{n=1}^{3} m=4,5,6\left|\tilde{x}\left(q_{0}-2, v_{0} \pm m, n\right)\right|^{2}+\left|\tilde{x}\left(q_{0}+2, v_{0} \pm m, n\right)\right|^{2}}
$$

On donne les résultats sur signaux réels du traitement comparativement au traitement actuel et deux de ses normalisations possibles ainsi que sur Pseudo-Wigner-Ville Lissée.

\section{CONCLUSION}

On pourra remarquer sur les résultats expérimentaux l'intérêt de la méthode présentée ici qui correspond à une démarche originale par rapport aux méthodes usuelles et aux grands axes de recherche en traitement du signal.

II nous reste encore à la qualifier par des mesures plus précises et à la calibrer avant de la rendre opérationnelle. Elle a été développée sur un array processor AP120 du Gerdsm. A ce propos, nous tenons à remercier M. Chr. PLUMEJEAUD qui a mis le calculateur et les bandes à notre disposition ainsi que M. A. MIALHE qui a codé les algorithmes sur l'AP. 


\section{BIBLIOGRAPHIE}

1. F. LefaudeuX - Chr. Plumejeaud - B. RAfine :

Algorithm de Normalisation (GRETSI 1981)

2. Chr. PLUMEJEAUD - J.Y. MORIN :

N.T./E.T.L.D/ GERDSM sur la Détection - Normalisation en Acoustique Passive (Traitement LOFAR)
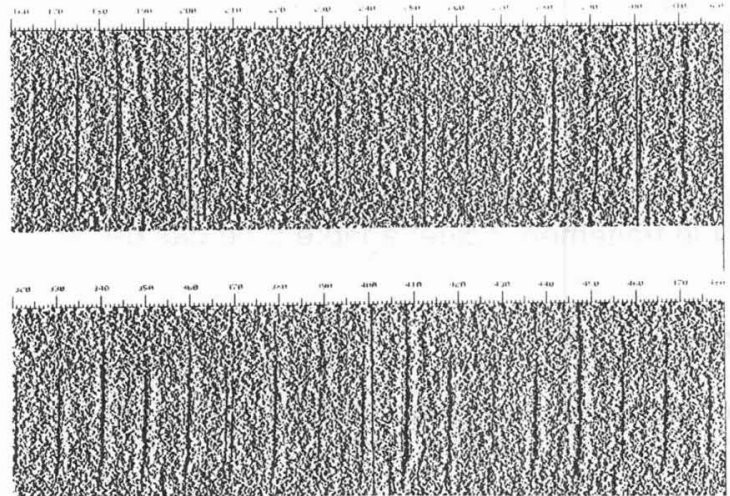

$1 a$
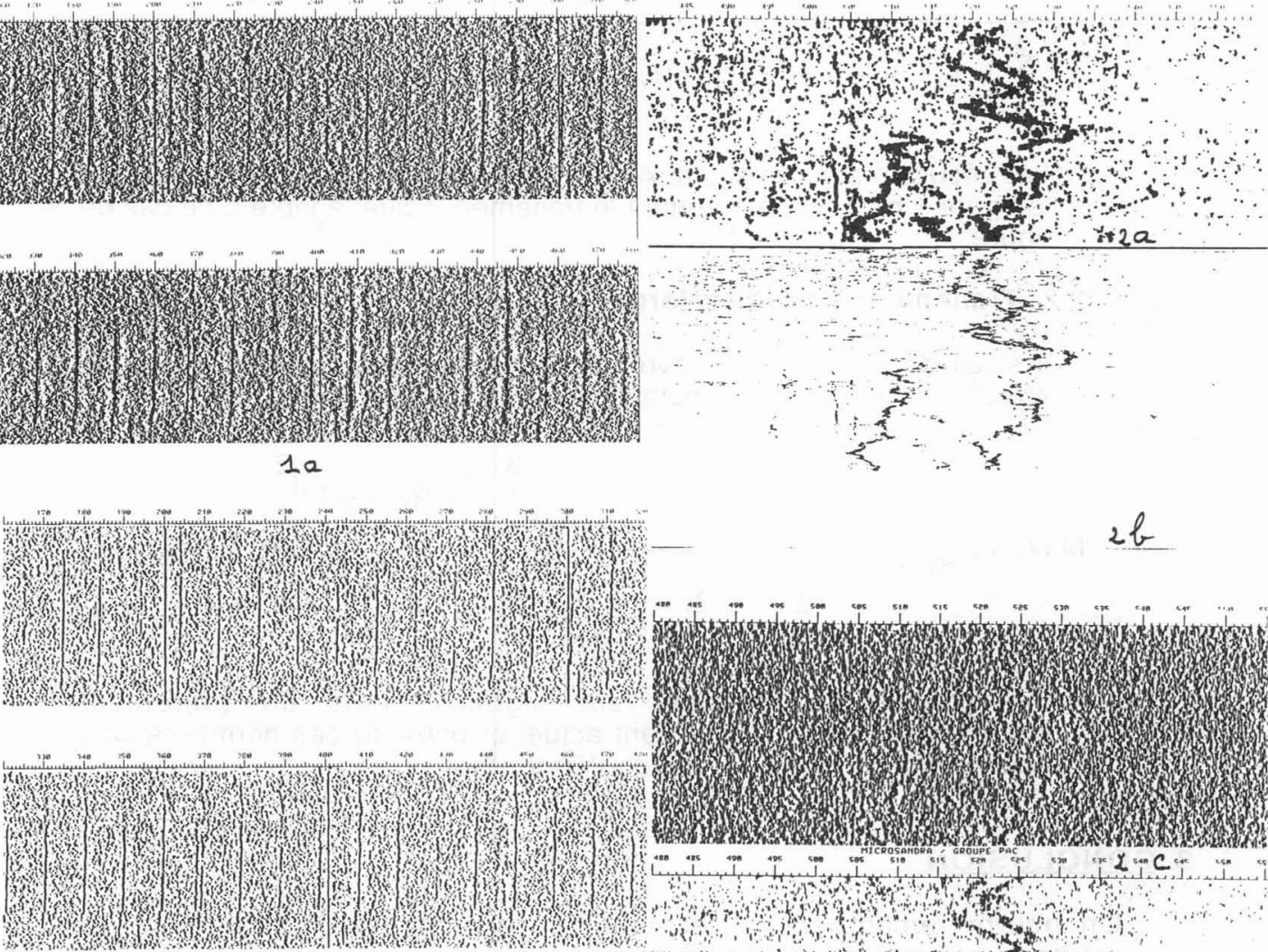

$1 b$

Figure 1b: Affinape Dopples/Figun 1s: ivartiment

Fily 2a: 7 weitiment 2D/R̈mis (anges \& flows

Figun ib: P WVL.

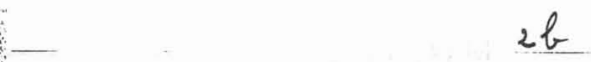

Figure 2c: initement otiual

Figure 2d: Trastoment otud/Nom flone

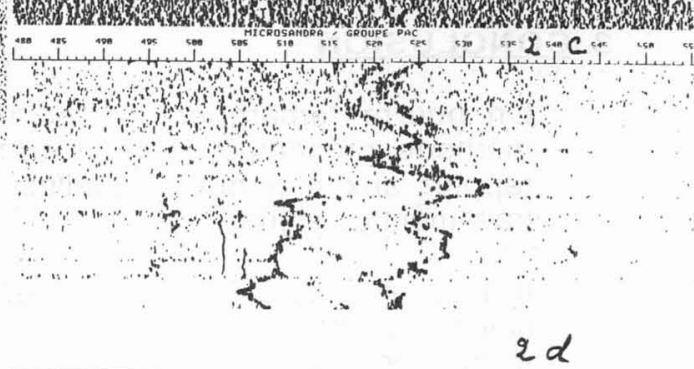

